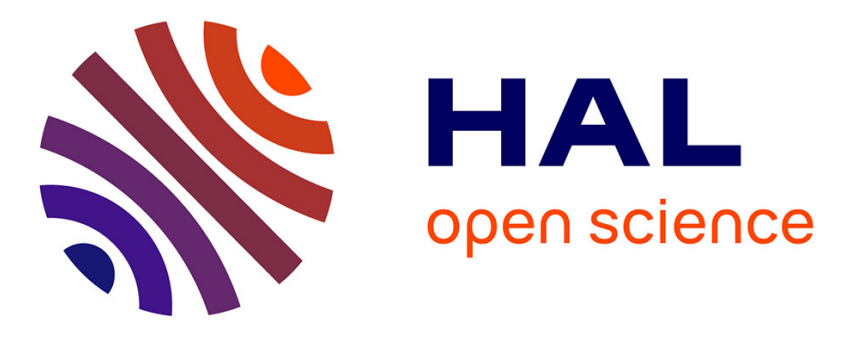

\title{
Passer les limites, rythmer le territoire. Paysage et mobilités du sanglier en Valbonnais (Isère, France)
}

Roméo Bondon, Raphäl Mathevet, Coralie Mounet, Simon

Chamaillé-Jammes

\section{- To cite this version:}

Roméo Bondon, Raphaël Mathevet, Coralie Mounet, Simon Chamaillé-Jammes. Passer les limites, rythmer le territoire. Paysage et mobilités du sanglier en Valbonnais (Isère, France). Géocarrefour Revue de géographie de Lyon, 2021, 95 (4), 10.4000/geocarrefour.16917 . hal-03223300

\section{HAL Id: hal-03223300 \\ https://hal.science/hal-03223300}

Submitted on 20 Oct 2021

HAL is a multi-disciplinary open access archive for the deposit and dissemination of scientific research documents, whether they are published or not. The documents may come from teaching and research institutions in France or abroad, or from public or private research centers.
L'archive ouverte pluridisciplinaire HAL, est destinée au dépôt et à la diffusion de documents scientifiques de niveau recherche, publiés ou non, émanant des établissements d'enseignement et de recherche français ou étrangers, des laboratoires publics ou privés. 
Titre : Passer les limites, rythmer le territoire. Paysage et mobilités du sanglier en Valbonnais (Isère, France)

Title : Pass the limits, give rhythm to the territory. Landscape and mobility of wild boar in Valbonnais (Isère, France)

Auteurs: Roméo Bondon, Raphaël Mathevet, Coralie Mounet et Simon Chamaillé-Jammes

Géocarrefour, 95/4, 2021

\section{Résumés}

Par la confrontation d'entretiens semi-directifs dans la vallée alpine du Valbonnais (Isère, France) avec l'observation de données de déplacements de sangliers, nous questionnons la contribution de la mobilité animale au devenir du paysage. Le sanglier se joue des limites administratives, révèle et anime les controverses spatiales et économiques des territoires humains, il pousse aussi, par sa biologie et ses spatialités à questionner les catégories d'analyse habituelles. Nous proposons de le qualifier $d$ ' « animal-frontière » et nous mettons en œuvre une approche à la fois interdisciplinaire et participative pour faciliter l'élaboration d'une connaissance située de l'espèce et des enjeux liés à ses déplacements. Nous explorons ainsi comment le sanglier participe d'un paysage vivant.

Our study takes place into the Valbonnais alpine valley (Isère, France) and is based on semistructured interviews which are confronted with the analysis of the movements of a local population of wild boars. We aim to question the contribution of animal mobility to the landscape dynamics. The wild boar interacts with administrative limits, reveals and stimulates the spatial and economic controversies of human territories, it also leads, by its biology and spatiality, to question the usual analytic categories. We suggest to understand wild boar as a « boundary-animal » and to implement an interdisciplinary and participatory approach to elaborate a situated knowledge of the species and the issues related to its movements, and thus to explore how wild boars shape a living landscape.

\section{Mots-clés}

géographie animale, faune sauvage, relation humains et animaux sauvages, chasse, sanglier, aire protégée

Keywords

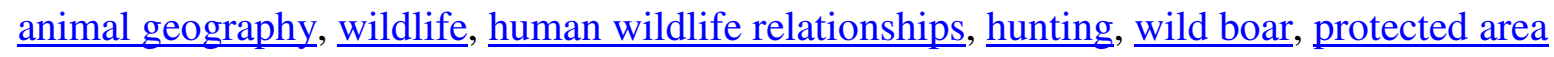

\section{Remerciements}

Nous remercions les élus, chasseurs, agriculteurs, habitants d'Entraigues et Valbonnais, les administrateurs et techniciens de la fédération départementale de l'Isère, ainsi que la direction et les agents du Parc National des Écrins pour leurs intérêts et accueil chaleureux pour cette étude. Ce travail est co-financé par la Fondation François Sommer, le Parc national des Écrins 
et le Syndicat Mixte des Gorges du Gardon, et co-piloté par Simon Chamaillé-Jammes et Raphaël Mathevet.

\section{Introduction}

Depuis la fin des années 1990, les animaux font l'objet d'une prise en compte croissante par les sciences sociales. La géographie n'a pas été en reste, et des travaux fondateurs en langue anglaise (Wolch et Emel, 1998 ; Philo et Wilbert, 2000 ; Whatmore, 2002 ; 2006) ainsi que, dans une moindre mesure, en français (Blanc et Cohen, 2002) ont ouvert deux décennies d'intense activité, sur laquelle des synthèses récentes sont utilement revenues (Buller, 2014 ; 2015 ; 2016 ; Hovorka, 2017 ; 2018 ; 2019 ; Gibbs, 2020a ; 2020b). S'il a été rappelé que la géographie avait par le passé montré un intérêt certain pour les animaux (Benhammou, 2016 ; Bortolamiol et al., 2017), ce n'était que de manière ponctuelle et mesurée, pour caractériser la répartition spatiale d'espèces données ainsi que l'évolution de cette distribution dans le temps - cette dimension, quoique marginale, perdure aujourd'hui, par exemple à propos de l'âne en France (Lompech et al., 2018). On peut actuellement distinguer trois axes principaux au sein d'une sous-discipline qui tend, autour de réseaux et concepts propres, à se structurer.

Le premier axe s'occupe des représentations que les humains se font des animaux, et comment ces dernières influencent les actions entreprises sur et avec les animaux. L'animal a dans ce cas une «valeur heuristique pour le géographe » (Blanc et Cohen, 2002, p. 32) permettant de mettre à jour la manière dont les humains appréhendent leur milieu de vie et quelle « juste place » ils accordent aux non-humains dans celui-ci (Philo et Wilbert, 2000 ; Mauz, 2002). Les représentations de certaines espèces, comme le castor, évoluent selon le temps long (Le Lay et al., 2017) ou les variations de dynamiques de population, comme pour la perruche à collier au sein de l'agglomération parisienne (Berthier et al., 2017). Le discours des acteurs tient alors une place prépondérante dans leur appréhension (Wolch et Emel, 1998), des méthodes tenant des sciences du langage pouvant dès lors être mobilisées (Chandelier, 2018).

Le deuxième axe est plus directement informé par la tradition géographique et se focalise sur les jeux d'acteurs suscités par la présence - ou l'absence - d'animaux sur un territoire donné. L'étude des conflits et controverses est privilégiée (Marchand, 2013). Si les prédateurs que sont le loup ou l'ours ont été tôt abordés (Benhammou, 2007 ; Mounet, 2007a) afin de caractériser les compositions et recompositions socio-spatiales auxquels ils participent, des espèces aussi diverses que le chevreuil (Boussin, 2008) ou les canards (Mathevet et al., 2003) ont pu être également étudiées.

Enfin, une troisième dimension tenant de la géographie culturelle essaie d'aller au-delà des représentations (Lorimer, 2006) afin de s'intéresser au processus relationnel complexe produit par le vivant (humain comme non-humain) au sein d'un paysage communément habité (Johnston, 2008). En plus des « animal spaces » attribués, volontairement ou non, par les humains, il s'agit d'explorer les « beastly places » des animaux étudiés, soit la manière dont ces animaux évitent, s'échappent, transgressent ou se jouent des espaces qui leur ont été attribués (Philo et Wilbert, 2000, p. 14). L'ontologie attribuée aux animaux dépend dès lors de l'échelle des relations qui se tissent, et de ce qui est privilégié en celle-ci (Lorimer et Srinivasan, 2013). Des études ont ainsi pu porter sur l'évolution de relations réciproques entre des éleveurs et leur bétail (Lorimer, 2006), sur des animaux singuliers selon une perspective biographique (Bear, 2011 ; Forsyth, 2016), ou encore sur la gestion du littoral et du flamant rose dans le contexte du ré-ensauvagement (Mathevet et Béchet, 2020), ou enfin les 
transgressions territoriales de mammifères en milieu rural, tels les éléphants (Barua, 2014), comme en milieu urbain, tels les macaques (Barua et Sinha, 2017).

Parmi ce bestiaire, le sanglier, dont la population est en France en constante augmentation depuis des décennies, figure au rang des « animaux à problème » étudiés par le deuxième courant (Mounet, 2007b ; 2011) en raison des dégâts qu'il occasionne aux espaces cultivés, des risques sanitaires et de collision routière qu'il représente, et de la difficulté à en assurer la gestion. Si tout un panel de solutions techniques est envisagé pour réduire sa population, des enjeux sociaux (partage de l'espace, usages et activités humaines contradictoires) et environnementaux (conservation, chasse, production agricole et forestière) participent à complexifier leur mise en œuvre au niveau local.

Pour appréhender ces enjeux et les mettre en dialogue, cet article propose une entrée sociospatiale se focalisant sur l'inscription des mobilités animales dans un paysage où se partagent, s'entremêlent et se superposent des spatialités humaines et non-humaines (Hodgetts et Lorimer, 2020). Nous proposons l'insertion de données écologiques issues de suivis GPS de sangliers, afin de participer d'une démarche pluridisciplinaire centrée non pas autour d'un seul corpus de notions, mais bien plutôt à la croisée d'un faisceau d'objets communs à la géographie et l'écologie spatiale, tentant d'ôter la « primauté de la discipline pour la rendre à l'objet »(Beck et Fabre, 2010, p. 20). Nous essayerons, à la suite d'appels déjà anciens (Thrift et Dewsbury, 2000 ; Rose et Wylie, 2005), de réinvestir et réanimer la notion de paysage telle qu'elle a pu être constituée jusqu'alors en nous arrêtant sur une espèce animale donnée (le sanglier) en un territoire circonscrit (une vallée alpine et ses versants).

Après avoir exposé le contexte, la problématique et la méthodologie, nous aborderons les manières dont les acteurs expérimentent les mobilités animales, depuis la connaissance spontanée de celles-ci jusqu'à la réception de leurs effets sur les activités humaines. Nous discuterons ensuite de la manière dont les sangliers, par leur mobilité, interrogent les frontières spatiales et symboliques des humains et participent ainsi de la fabrication d'un paysage dans lequel ils sont pleinement inscrits. Nous analyserons comment le croisement de connaissances scientifiques et locales sur ces mobilités peut lui-même participer à dynamiser les jeux d'acteurs et transformer les situations locales.

\section{$\underline{\text { Faire cas des sangliers }}$}

\section{Contexte}

Le sanglier (Sus scrofa, L. 1758), ongulé artiodactyle de la famille des suidés, est une espèce ubiquiste que l'on rencontre dans tous les milieux, en plaine comme en montagne (Baubet, 1998). Sa population de plus en plus abondante depuis la seconde guerre mondiale accroît les effets écologiques (Massei et Genov, 2004 ; Vallée et al., 2016), sociaux et économiques (Mounet, 2007a) de l'espèce. Cette démographie s'explique par la prolificité des laies, l'augmentation des surfaces messicoles et les évolutions climatiques (Vetter et al., 2015), mais aussi par les pratiques cynégétiques dites «conservatrices »- sélection dans les tirs, agrainage $^{1}$, élevage (Baubet 1998).

\footnotetext{
${ }^{1}$ L'agrainage est un terme cynégétique, employé à des fins de gestion, consistant en la mise à disposition pour le gibier de nourriture, généralement de maïs égrainé, dans le but d'attirer des populations locales dans un lieu particulier. L'agrainage est dit dissuasif quand il permet d'éloigner les animaux d'une culture vulnérable en les maintenant à l'écart de celle-ci.
} 
Le régime alimentaire et la grande capacité d'adaptation du sanglier en font une espèce opportuniste (Ballari et Barios-Garcia, 2014 ; Bobek et al., 2017). En certains endroits, cultures et jardins deviennent des sites de nourrissage privilégiés. Les dégâts que les sangliers y occasionnent, auxquels il convient d'ajouter les risques de collisions et les enjeux sanitaires liés à certaines maladies dont ils peuvent être porteurs, entraînent des réactions sociales (conflits d'acteurs sur les effets produits par cette espèce), politiques (discours antagonistes sur la nature de l'espèce) et juridiques (production de lois et décrets pour organiser la gestion de l'espèce).

\section{À tout problème existe sa solution...}

En l'absence de prédateurs naturels en nombre suffisant, en raison d'une intense pression humaine sur les espèces et leurs habitats, la gestion des populations de sangliers échoie historiquement à la chasse. La principale réponse apportée par les responsables cynégétiques, les gestionnaires d'aires protégées ou les biologistes et écologues, passe ainsi par une augmentation des prélèvements de sangliers par les chasseurs (Tolon et al., 2009 ; Thurjfell et al., 2013 ; Massei et al., 2015 ; Quiros-Fernandez et al., 2017).

Depuis 1968, les fédérations départementales des chasseurs indemnisent les dégâts subis et déclarés par les agriculteurs, en échange du retrait du droit d'affût de ces derniers (Charlez, 2008). Mais ce compromis semble aujourd'hui trouver ses limites : les réactions politiques actuelles - rapport parlementaire, réunion entre syndicats d'agriculteurs et fédérations de chasseurs - en constituent un symptôme incontestable. Les conflits se sont multipliés sur le territoire national et persistent toujours malgré les orientations gestionnaires impulsées dans les départements et le cadre d'actions technique proposé par l'État en 2009 avec le plan national de maîtrise du sanglier (PNMS). Bien que la gestion concrète des populations soit établie au niveau départemental par les différentes fédérations départementales des chasseurs, en accord avec le préfet et les services de l'État et en concertation avec les institutions et acteurs concernés, sa mise en œuvre concrète dépend des pratiques locales des chasseurs, elles-mêmes enchâssées dans des configurations environnementales et sociales particulières (Mounet, 2007a).

Or, si la pratique de la chasse au sanglier a pu être étudiée selon des approches anthropologiques dans le nord-est de la France (Hell, 1998), les Cévennes (Pelosse et Vourc'h, 1982 ; 1984 ; Pelosse, 1993), ou selon des approches géographiques dans le sud (Raynal, 2004) et le sud-ouest de la France (Saldaqui, 2013), peu d'études englobent l'ensemble des acteurs mobilisés par les effets de l'espèce (Mounet, 2007 ; 2011 ; Mathevet et al. 2018) et les comportements situés des populations de cette même espèce. Outre les chasseurs, les agriculteurs, les particuliers, les forestiers, les gestionnaires d'espaces protégés et les services administratifs dédiés sont concernés par les agissements des sangliers et participent du contexte d'action local autour de sa gestion.

\section{$\underline{\text { À toute solution peut surgir un nouveau problème }}$}

De nombreuses mesures techniques sont envisagées pour réduire les populations, tout particulièrement dans des lieux potentiellement conflictuels, identifiés comme «points noirs sangliers »(PNMS, 2009). Mais la multiplication de ces mesures entre en confrontation avec leur réalisation locale. Les intérêts politiques nationaux, cynégétiques départementaux et ceux des chasseurs à l'échelle de leur territoire de chasse ne sont pas nécessairement équivalents. Certains chasseurs se limitent parfois dans leurs pratiques pour maintenir une population de 
sangliers favorable à la chasse ; ils doivent composer avec des relations souvent conflictuelles et concurrentielles entre équipes de chasse ; ils s'insèrent, enfin, dans des jeux d'acteurs locaux qui dépassent dans certains cas la problématique du sanglier (Mounet, 2011). En somme, l'ensemble de ces intérêts locaux peuvent participer d'une pression de chasse atténuée. Le déclin du nombre de chasseurs en activité et l'augmentation de leur moyenne d'âge participent également de cette dynamique, et ce dans toute l'Europe (Massei et al., 2015). Les chasseurs ont par ailleurs à mettre en œuvre une régulation tout en composant avec les particularités de leur territoire : la géographie des lieux, de même que la réglementation (réserves de chasse, aires protégées) peuvent limiter voire interdire l'accès à une partie du territoire dont ils sont responsables. Ajoutons à cela l'incertitude relative aux comportements et mobilités des sangliers, bien que ces derniers soient de plus en plus étudiés (Morelle et al., 2015).

Ainsi, les incertitudes qui caractérisent de telles situations locales relèvent d'un double registre : la connaissance des mobilités animales ne peut être considérée sans la compréhension des jeux d'acteurs influençant les conflits sociaux et les postures de chacun. Il s'avère donc nécessaire de combiner entre elles des disciplines autonomes - sciences naturelles, sociales et humanités - avec les savoirs locaux et vernaculaires récoltés.

\section{$\underline{\text { Problématique }}$}

Quelle que soit la problématique ou l'échelle étudiée à propos des effets des sangliers, la mobilité de l'espèce en constitue une des causes premières.

\section{Mobilité spatiale et conceptuelle : déplacements disciplinaires}

La récente unification des travaux sur la mobilité animale dans la sous-discipline de la movement ecology souligne l'intérêt croissant portés par les écologues aux déplacements animaux (Nathan et al., 2008). Ces derniers sont en effet particulièrement altérés par les changements environnementaux et paysagers actuels (Tucker et al., 2018). S'il apparaît que la répartition de la nourriture disponible et les conditions d'accès à celle-ci définissent en grande partie les déplacements que vont effectuer les sangliers (Morelle et al., 2015), la chasse modifie également le comportement spatial de l'espèce (Toïgo et al., 2008 ; Thurfjell et al., 2013 ; Fattebert et al., 2017). L'investissement de la dimension spatiale des comportements animaux par l'écologie incite la géographie à se saisir des résultats avancés pour apporter un éclairage supplémentaire à ses propres études. Si les notions employées peuvent être similaires - territoire, ressource, paysage - le sens qui leur est attribué n'est pas tout à fait partagé. Un travail d'unification conceptuelle autour de l'objet considéré est donc à entreprendre. Ainsi, aux trois courants de la géographie animale définis précédemment, un quatrième peut être ajouté à l'intersection de l'écologie spatiale et de la géographie. La mobilité des animaux peut alors servir d'entrée innovante, ajoutant au suivi spatial « les trajectoires et expériences vécues par les animaux » (Hodgetts et Lorimer, 2020, p. 2). Approches écologique et socio-spatiale se complètent dès lors dans l'étude des relations qu'entretiennent les animaux avec leur milieu et les sociétés humaines environnantes.

Il nous semble que les sangliers sont à même d'interroger tant la gestion dont ils font l'objet, que celle des aires protégées qu'ils usent et traversent. La consolidation de la notion d'« effet réserve » au sein de la littérature gestionnaire (Tolon et Baubet, 2010) inclut dans le questionnement ces espaces non-chassables dans lesquels les sangliers sont accusés de se réfugier pour en sortir nuitamment, contraignant dans ce cas l'efficacité de leur gestion à leur 
périphérie. La dimension spatiale de la conservation est alors à mobiliser (Mathevet et Godet, 2015). Par ailleurs, les sangliers déjouent par leur mobilité les représentations usuelles de la grande faune et de ses comportements - sauvage, domestique, artificiel, naturel. Il convient donc d'aller par-delà les représentations sans pour autant les nier (Lorimer, 2006), afin de comprendre pourquoi une espèce telle que le sanglier échappe à une catégorisation figée. Nous proposons le concept «d'animal-frontière », postulant que c'est parce qu'il se situe luimême à l'intersection de différentes catégories biologiques, spatiales, et représentations anthropologiques que les frontières se meuvent en même temps qu'un individu donné, voire qu'une population donnée de cette espèce. Les mobilités des sangliers interviennent dans des paysages particuliers, et participent à la co-produire.

\section{Mobilité animale et réanimation du paysage}

L'étude des sangliers permet selon nous de participer à cette « réanimation » que d'aucuns ont pu réclamer à propos de la notion de paysage (Rose et Wylie, 2006). Héritant d'un cadrage géographique ni entièrement constructiviste, ni seulement matérialiste qui postule une influence réciproque du milieu et de ses habitants (Bertrand, 1978 ; Berque, 2000), nous le réinvestissons en nous inspirant d'une anthropologie qui prend en compte un enchevêtrement d'usages humains et non-humains (Ingold, 1993 ; Janowski et Ingold, 2016) sans ignorer les approches écologiques dont a fait l'objet la notion de paysage (Girel, 2006). Par là, nous souhaitons réinsérer les animaux, et en particulier les sangliers, dans la compréhension de paysages qui, sans eux, lorsque le vivant n'est que végétal ou humains, nous semblent incomplets. L'interdisciplinarité de cette étude invite à une appréhension renouvelée des approches et concepts de la géographie animale.

S'il est évident que les manières humaines d'habiter un lieu participent de la formation spontanée du paysage, leur dimension territoriale implique une relative fixation de certaines de ses portions, tant sur le plan des représentations que matériellement. Les limites administratives et symboliques ont une matérialité territoriale certaine, qu'il convient de décrire, comprendre et étudier. Nous souhaitons leur confronter le point de vue des sangliers, ou, du moins, leurs usages du paysage et leurs mobilités au sein de celui-ci. Les imprévisibles déplacements des sangliers déforment et malmènent certaines limites humaines, apportant un trouble supplémentaire au paysage ; une attention forte à l'égard de ses déplacements participe d'un paysage dont la perception est vivifiée. Les traces matérielles (Gagnol et al., 2018), les suivis cynégétiques, sanitaires ou scientifiques, les témoignages et les réactions que suscitent les sangliers et leurs déplacements offrent un regard différent de celui proposé par les textes réglementaires et les pratiques cloisonnées des différents acteurs; leur confrontation par le croisement de méthodes scientifiques et des savoirs vernaculaires permet-elle une meilleure efficacité des débats locaux ? Permet-elle d'aboutir à une connaissance située et plurielle des manières dont les sangliers s'inscrivent dans le paysage ? Qu'implique et révèle dès lors ce travail d'inscription des sangliers dans le paysage ? Si ce dernier est animé par des agissements et usages humains et non-humains qui sédimentent avec le temps, au point de former ce que Tim Ingold a appelé un «taskscape », qu'ajoute la prise en compte spécifique des sangliers dans celui-ci ? Comment les mobilités de cet animal révèlent les tensions entre humains et non-humains et interrogent les mobilités et fixités humaines de ce «taskscape »? Comment les usages se retrouvent-ils affectés ? Cela peut-il conduire à la requalification du sanglier comme un « animal-frontière », portant en lui des limites considérées usuellement comme paysagères mais dès lors en mouvement avec les différents individus et populations ? 


\section{Terrain et méthode}

Nous examinerons ces questions à partir des résultats d'une étude de terrain réalisée de février à août 2019, principalement en-dehors de la saison de chasse, dans la vallée du Valbonnais (Figure 1), au sein du massif des Écrins, en Isère et plus particulièrement sur la commune d'Entraigues. Cette dernière comptait 230 habitants en 2017 et accueille le siège du secteur du Parc national des Ecrins (PNE). En effet, si le Valbonnais est une petite région historique de la vallée de la Bonne, au sud de l'Isère, ainsi qu'un ensemble de communes groupé autour d'un village éponyme, c'est également un secteur de la zone cœur du PNE dont les limites ne recoupent que partiellement celles des communes concernées. Créé en 1973, le PNE est depuis un acteur majeur du territoire. La chasse est interdite dans sa zone cœur, interdiction en partie à l'origine des conflits concernant les sangliers dans ce territoire.

Cette étude fut mise en œuvre dans le cadre d'un stage s'inscrivant dans un projet mené sur trois ans par le CNRS (CEFE, UMR 5175, Montpellier) dans les Écrins et le Gard. Les objectifs de ce projet de recherche, dont nous relatons ici la première année de mise en œuvre, sont d'analyser la mobilité des sangliers par colliers GPS et pièges photographiques, ainsi que de mettre en place et animer un dialogue inter-acteurs (agents du PNE, agriculteurs, chasseurs, habitants, forestiers) autour de ces données et de leurs propres expériences. Le déploiement de colliers GPS a permis et permettra de proposer à la discussion des résultats tenant de l'écologie des déplacements et de quantifier les usages que font les sangliers du paysage. Pendant la période étudiée, un mâle de $80 \mathrm{~kg}$ et une femelle de $60 \mathrm{~kg}$ ont été capturés et équipés. Des pièges-photographiques utilisés en mode vidéo (de 11 à 25 au cours de la période) et disposés à la frontière de la zone cœur et de l'aire d'adhésion du PNE au niveau du village d'Entraigues permettent également d'y documenter les déplacements animaux.

Figure 1 : Localisation des communes du Valbonnais (Isère, France) et de la zone cœur du Parc National des Ecrins. Sources : IGN, PNF 


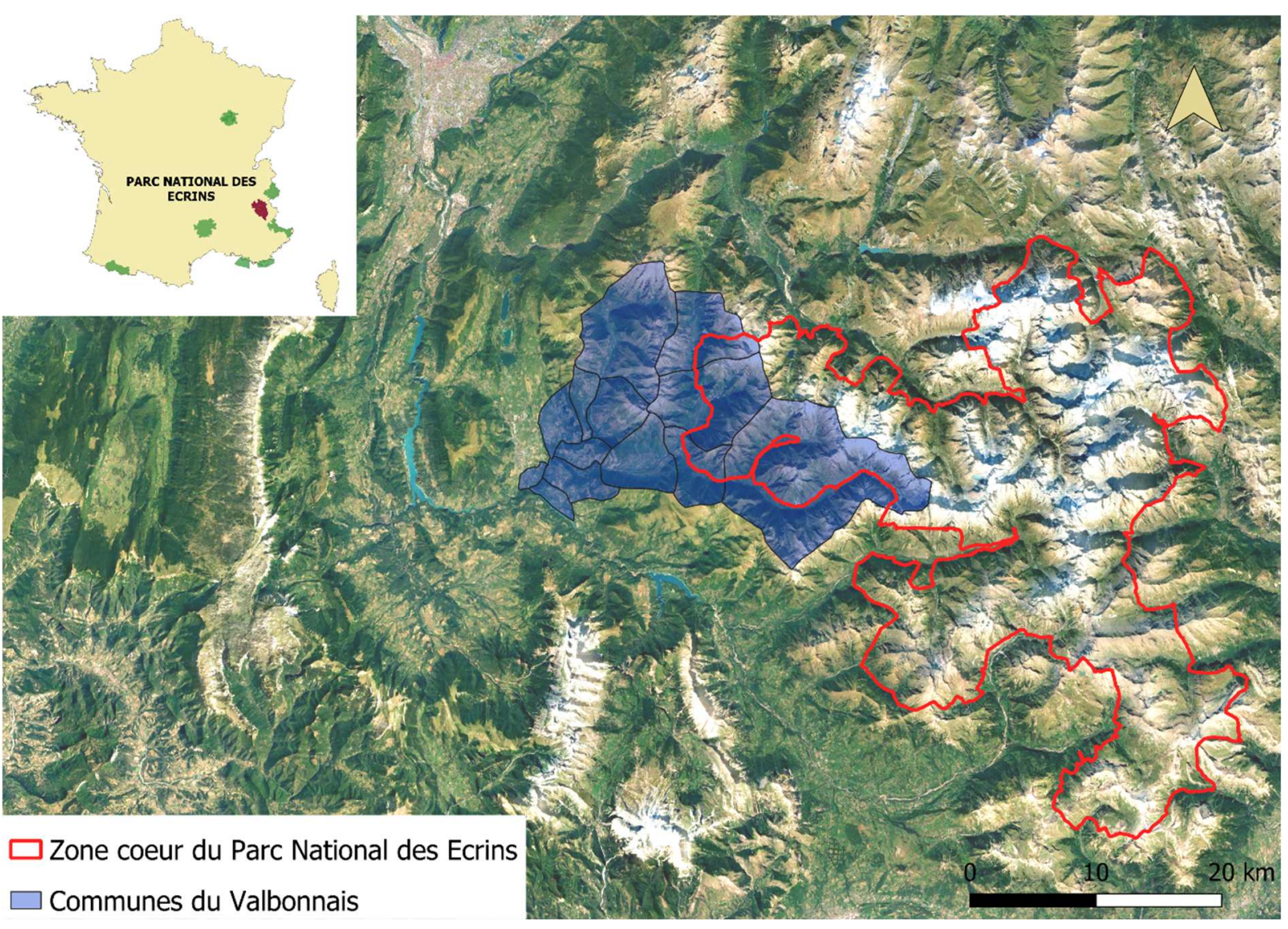

Le projet a pour ambition de démontrer la possibilité de faire coopérer l'ensemble des acteurs pour définir et évaluer des mesures de gestion, en s'appuyant sur une étude des déplacements de sangliers. Une telle étude offrira des données objectives et des objets autour desquels articuler la discussion. Le concept d'objet-frontière (Star et Griesemer, 1989) souligne le rôle de certains artefacts (ici le collier GPS, les photos issues de pièges-photographiques et les cartes des déplacements des animaux) qui interviennent dans l'élicitation, la collecte, la gestion et la coordination de connaissances distribuées. Ces objets-frontières servent de supports de traduction entre experts et non-experts, et interviennent ainsi comme supports d'intégration de la connaissance située et supports de médiation sociale. L'objet-frontière devient objet-intermédiaire (au sens de Vinck, 1999) lorsque l'on s'intéresse, comme ici, au processus de stabilisation des faits dans un collectif, ainsi qu'au processus de médiation entre différentes visions et connaissances du monde. La confrontation de ces objets-frontières avec le discours des différents acteurs, ainsi que le récit de leur pratique du paysage en relation avec les sangliers nous permettront de lier les animaux avec ses fonctions et effets au sein de ce même paysage.

Ces discours ont été recueillis au cours de 21 entretiens semi-directifs conduits auprès des acteurs représentés ci-dessous (Tableau 1). La sur-représentation de chasseurs ou d'anciens chasseurs (aucune femme chasseresse n'a été interrogée) tient à la problématique étudiée, à l'intérêt manifesté pour celle-ci et à un nombre plus grand de représentants ; leur nombre est plus équilibré avec le reste des personnes enquêtées si l'on ne considère que les membres actifs de l'Association communale de chasse agréée (ACCA) d'Entraigues, site principal de l'étude.

Tableau 1 : Les différents groupes définis préalablement à l'enquête dans lesquels se situent les acteurs interrogés. Source : les auteurs, enquête 2019 


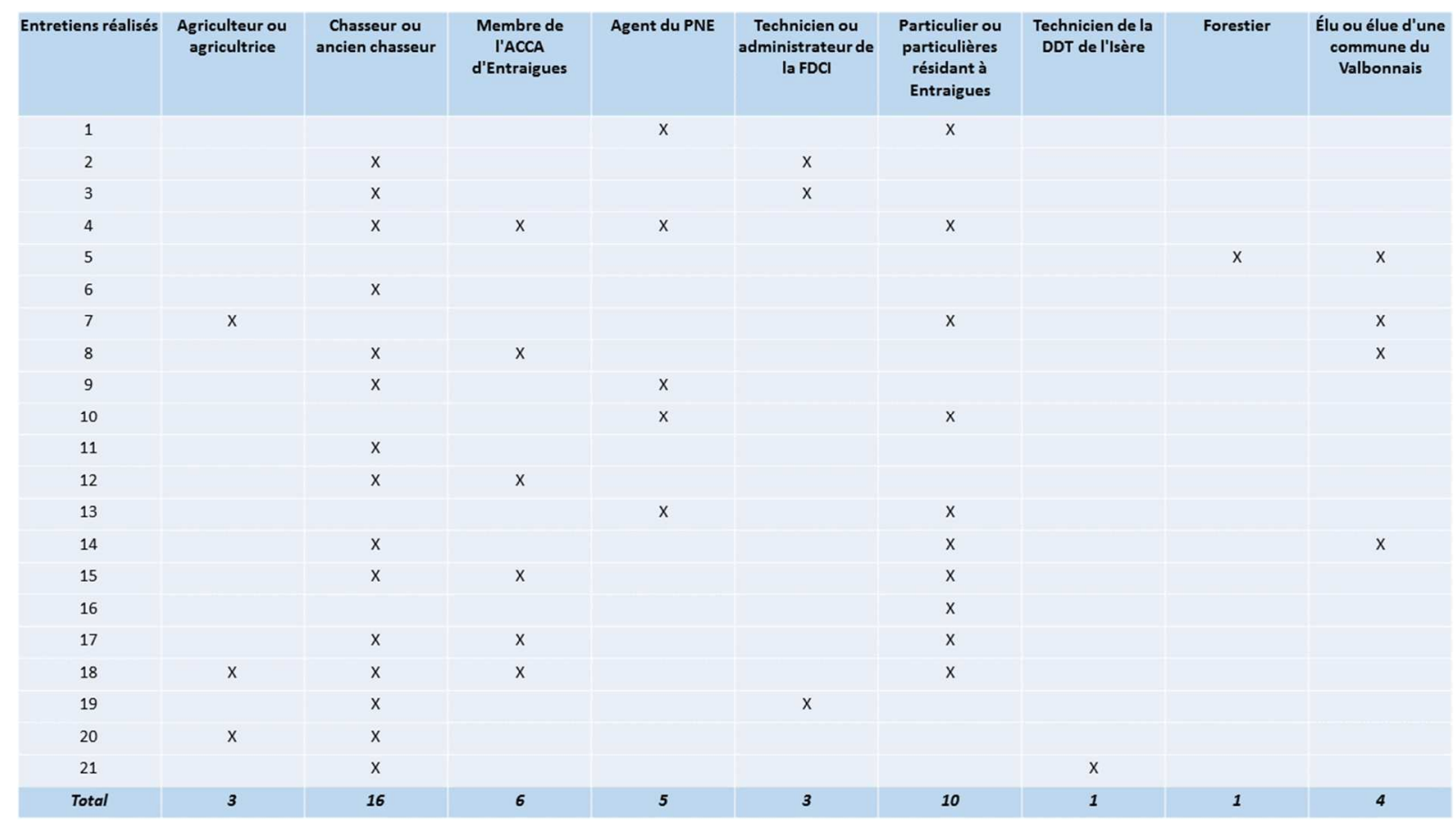

Les entretiens enregistrés et intégralement retranscrits, ont été analysés selon une méthode qualitative (De Sardan, 2004). De nombreuses rencontres les ont complétés. Celles-ci ont consisté en la participation à des réunions formelles et informelles entre chasseurs, à la fréquentation quotidienne d'agents du parc du secteur lors des sessions de terrain, ainsi qu'à l'organisation de trois réunions et d'un atelier avec les acteurs concernés. Entretiens, observations et captures ont donc été pour la plupart mis en œuvre au sein et autour du village d'Entraigues. Afin d'éviter que l'étude ne privilégie le côté humain des relations interspécifiques (Seymour et Wolch, 2010, p. 306), il a été choisi de croiser les résultats issus de telles méthodes avec le suivi spatial d'individus et d'une population.

Le dépouillement des vidéos enregistrées par les pièges-photographiques a permis de se familiariser avec la faune locale, et les sangliers en particulier. Les représentations de trajets GPS de deux sangliers ont été discutées collectivement lors d'une réunion publique avec certains des acteurs précédemment cités et interrogés, ainsi qu'en présence des scientifiques pilotant le projet. À partir des discours recueillis et des activités observées, se dessinent les formes de la perception qu'ont les habitants des spatialités des sangliers avec lesquels ils sont en contact.

Nos premiers résultats, issus de la confrontation de ces différentes méthodes, seront discutés à partir d'un corpus tenant tant de la géographie des animaux que de l'écologie spatiale et de l'anthropologie, afin de reprendre les questions énoncées et de caractériser la part que peuvent occuper certains animaux à propos de la représentation et des usages d'un paysage donné.

\section{$\underline{\text { Résultats : faire l'expérience des spatialités des sangliers }}$}

\section{Les dégâts, matrice d'une rencontre médiatisée}

Le village d'Entraigues, principal site de l'étude, se situe à la confluence de deux vallées torrentielles (Figure 1). Dans son dos - ou en face du village selon le point de vue - la vallée de la Bonne. De l'autre côté, un versant abrupt qui marque les débuts de la zone cœur de PNE. Entre la zone bâtie et la pente, une plaine mise en culture ou en pâture marque une transition. 
C'est sur cette plaine que se concentrent les dégâts subis par les agriculteurs ou les habitants, et c'est dans le versant la surplombant que les sangliers, selon la totalité des chasseurs et certains particuliers et gestionnaires interrogés trouveraient refuge.

La somme des dégâts causés par les sangliers en France est avancée comme preuve d'une surpopulation délétère pour le monde agricole. Les indemnisations pour tout gibier s'élevaient à 37 millions d'euros à l'échelle nationale pour la saison 2016-2017 (Cardoux et Perea, 2019); en moyenne 400000 euros pour le département de l'Isère. Le montant des indemnisations délivrées par les fédérations des chasseurs aux agriculteurs ne font qu'augmenter depuis les années 1970 (Carni et Fachinni, 2012), ce que les administrateurs et techniciens de la Fédération des chasseurs de l'Isère (FDCI) nous ont tous confirmés. Si des réactions juridiques et politiques sont mises en œuvre à l'échelon national afin de compenser les pertes et limiter les effets des sangliers localement, «c'est le niveau départemental avec la fédération des chasseurs qui constitue l'échelle décisive » (Mounet, 2007a, p. 129). Récipiendaire des demandes d'indemnisation de la part des agriculteurs touchés par des dégâts et outil de gestion des populations, la FDCI, comme les autres fédérations départementales, doit allier une réponse efficace à la demande de régulation et assurer des effectifs suffisants de gibier pour ses adhérents. Ce double-jeu mène à des incompréhensions, voire des conflits entre monde cynégétique et agricole à l'échelle locale comme départementale, acteurs auxquels il faut ajouter des particuliers au jardin retourné ou des gestionnaires d'aires protégées.

La prise en compte des dégâts oriente la définition de la controverse locale en fonction du contexte de leur découverte, de leur répétition et des répercussions économiques et sociales qu'ils peuvent avoir sur les personnes concernées. Un habitant de sensibilité naturaliste pourra les minimiser, d'autant plus si les victimes n'ont pas d'usage productif de leur terrain :

«Quand on habite en pleine montagne, quand c'est juste un gazon retourné, pour moi c'est quand même des dégâts un peu à la marge... Des maisons secondaires, ils devraient peut-être être contents de voir des sangliers de leurs fenêtres ${ }^{2}$. »

Les paysans, à l'inverse des particuliers, sont unanimement reconnus comme les premiers touchés par les effets des sangliers. Si la surface concernée et indemnisée à Entraigues est variable (plus de six hectares en 2018, moins de un la plupart des autres années, pour des montants indemnisés pouvant aller jusqu'à 4000 euros), la charge de travail agricole imposée par des parcelles que les reliefs restreignent légitime selon la majorité des enquêtés la colère des agriculteurs - envers les populations locales de sangliers comme envers d'autres acteurs. La difficulté tient dès lors à trouver des responsables humains pour canaliser les griefs et des moyens de limiter les dégâts.

Le télescopage de réactions politiques et techniques à l'échelle départementale et locale implique des discours structurants qui diffèrent selon les niveaux de gestion. Néanmoins, quel que soit l'outil réglementaire convoqué par des chasseurs, les aires protégées sont stigmatisées. Les «points noirs » issus d'un plan national en sont le meilleur exemple, comme l'illustre ce technicien de la FDCI :

\footnotetext{
${ }^{2}$ Entretien avec un forestier et riverain, 3 avril 2019
} 
« Nous, fédération, notre politique c'est que toute zone non chassée jouant un effet réserve est pour nous un point noir. Parce que [...] dans ces zones les sangliers vont se réfugier, ne pourront pas être régulés et, après la chasse, pourront ressortir et faire des dégâts ${ }^{3}$. »

Dans les communes ayant une partie de leur finage dans la zone cœur du PNE, les équipes locales qui chassent les sangliers portent ce même discours. Les chasseurs d'autres gibiers apportent des nuances par rapport à leurs espèces de prédilection, en particulier les chamois : pour certains, les populations auraient bénéficié du zonage de protection. Le consensus entre fédération et chasseurs locaux se fait plus fragile lorsqu'il s'agit des modes de gestion envisagés. Celui-ci s'applique à l'ensemble du territoire national, mais est adapté en fonction des spécificités des départements et, pour certaines espèces, des spécificités locales. La volonté nationale et départementale de réduire les populations de sangliers conduit à un allongement de la saison de chasse, à des aménagements pour permettre des tirs individuels ou des battues anticipées, et à supprimer certaines conditions contraignant jusqu'alors la pratique. Ces dispositions sont toutefois discutées par les ACCA qui reprennent réglementairement, mais aussi tacitement, les dispositions des échelons supérieurs.

33Ainsi, la pression de chasse ne dépend pas seulement du nombre de sangliers présents sur le territoire à disposition des pratiquants, mais aussi de la composition des équipes locales, des traditions de chasse ou de l'histoire cynégétique de chaque commune. Les clivages macrosociaux concernant les sangliers ne se recoupent pas nécessairement à l'échelle communale : des recompositions et « arrangements locaux » sont à l'œuvre (Mounet, 2011, p. 92).

Les groupes locaux identifiés au début de l'enquête ne sont en effet pas homogènes. Si les agents du PNE sont relativement en accord, la proximité que certains entretiennent avec la chasse modère leur défense stricte de la « réponse graduée ${ }^{4} »$ produite par le conseil scientifique du PNE. De même, l'appréhension des sangliers par la profession agricole dépend des activités cynégétiques pratiquées ou non par ses représentants. Parmi nos rencontres, aucun profil ne s'est répété : alors qu'un couple d'éleveurs avec des terrains fréquemment retournés s'en prend indifféremment aux représentants du PNE et aux chasseurs locaux, un éleveur également chasseur de chamois défend une pratique régulatrice envers les sangliers qui touchent ses pâturages, pratique dont il se charge. Enfin, un dernier éleveur, dont la coupe et la vente de bois complètent l'activité principale, paraît moins en difficulté avec l'espèce, et pour cause : il fait partie d'une équipe chassant en battue le sanglier et ne cache pas le souhait, partagé avec ses camarades, de maintenir une population élevée.

35Des alliances locales varient donc en fonction du contexte - dégâts élevés ou non -, des moyens proposés par les uns et les autres pour limiter les effets négatifs des sangliers et de l'écoute qui est apportée par les principaux responsables désignés. Les chasseurs - FDCI et locaux - et le PNE sont toutefois mis en cause sur des points précis qui cristallisent les débats : la zone cœur du Parc et l'agrainage.

\footnotetext{
${ }^{3}$ Entretien avec un technicien de la FDCl, 22 mars 2019.

${ }^{4}$ La « réponse graduée » est la doctrine du PNE en cas de dégâts à cause de sangliers sur les communes de la zone cœur. Elle liste un ensemble de mesures graduelles à prendre en fonction de la situation. Ses mesures engagent dans un premier temps les chasseurs et agriculteurs, et en dernier recours peut entraîner une inflexion temporaire de la réglementation du PNE.
} 


\section{$\underline{\text { Dépasser les controverses sociales et spatiales }}$}

Souvent abordée de manière spontanée au cours des rencontres, la pratique présumée ou avérée de l'agrainage divise entre non-chasseurs et chasseurs, et au sein même de l'activité, entre ceux qui chassent exclusivement les sangliers et ceux dont ce n'est pas la spécialité. Déjà abordée dans la littérature (Mounet, 2007 ; 2011 ; Raynal, 2004), nous choisissons ici de reléguer la question des effets de l'affouragement des animaux sauvages au second plan pour insister sur le débat vif que constitue le sort d'une espèce gibier entre enjeu cynégétique et dispositif spatial de protection du milieu naturel.

Le PNE, par la définition d'un espace non-chassable en sa zone cœur, ajoute des tensions supplémentaires aux sources de discorde classiques décelables en d'autres territoires, et particularise la controverse pour les communes proches du Valbonnais sur les enjeux de la protection et de la chasse. Les expressions d'« effet réserve » et de «zone refuge » mériteraient une généalogie à part entière. On peut toutefois noter en guise d'esquisse qu'elles sont nées dans le domaine de l'écologie marine, avant d'être employées dans les expertises gestionnaires, pour être reprises ensuite dans des travaux scientifiques. Leur emploi n'étant pas normalisé, et l'instabilité de ces termes peut être source de confusion voire de controverses.

Pour les techniciens et administrateurs de la FDCI, l'« effet réserve » est un poncif depuis longtemps prouvé. La convocation de la littérature gestionnaire et d'exemples dans le reste du département leur servent de références pour confirmer cette assertion - et délégitimer, parfois, la pertinence d'une nouvelle étude, à l'instar de ce technicien de la FDCI :

«C'est des études qui ont déjà été faites et refaites, ce que vous allez faire ça a déjà été fait ailleurs, c'est pas nouveau, à chaque fois ça a montré la même chose, c'est que le sanglier c'est une espèce très adaptative, hors période de chasse il va utiliser [...] une majorité des territoires sur lesquels il pourra trouver du gîte, du couvert et de la bouffe, et en période de chasse il va chercher des zones de tranquillité, des zones où il va pas se faire tirer dessus ${ }^{5}$. »

Pour leur part, si les agents du PNE ne remettent pas en doute la pertinence d'un effet produit par l'interdiction de chasser en zone cœur, ils en tempèrent l'importance comme cause de l'évolution démographique des populations locales de sangliers. Autour d'un cœur nonchassable s'étend en effet une zone de chasse sans commune mesure sur le plan territorial. Ainsi questionnent-ils l'effort de chasse réellement mené par les chasseurs locaux, et la pression plus ou moins forte exercée sur les individus :

«On a nettement eu l'impression ces dernières années que les chasseurs n'ont pas insisté autant qu'ils auraient pu faire sur les prélèvements et autant que c'était demandé par les agriculteurs surtout ${ }^{6}$. »

Commentant l'étude proposée, certains ne cachent pas, carte du secteur à l'appui, leur espoir de voir les trajets GPS invalider l'« effet réserve » de la zone cœur du parc. En effet, si le versant à l'est d'Entraigues concentre l'attention en raison de son statut de protection, celui au

\footnotetext{
${ }^{5}$ Entretien avec un technicien de la FDCl, 22 mars 2019.

${ }^{6}$ Entretien avec un agent du PNE et riverain, 20 mars 2019.
} 
nord du village présente les mêmes dispositions géographiques, laissant croire que les sangliers pourraient fréquenter l'un autant que l'autre.

Par ailleurs, l'observation d'habitants infirme les dires publics des chasseurs de sangliers « on n'a pas entendu beaucoup tirer » rappelait en réunion une agricultrice - ainsi que le tableau de chasse local. C'est aussi le cas parmi les chasseurs extérieurs à l'équipe dédiée aux suidés :

« La grosse équipe qui va aux sangliers chez nous là, de l'ouverture de la chasse en septembre jusque 28 février, ils ont fait douze battues; donc c'est zéro, c'est nul. C'est nul. C'est pas assez ! C'était ouvert tous les jours sauf le vendredi hein. Et à Entraigues il s'est tué douze, douze $^{7} \ldots »$

Auprès des chasseurs locaux, l'argument de l'« effet réserve » n'est convoqué qu'à la marge tant il semble évident pour qui connaît le terrain. S'il est employé, il ne constitue par le cœur du problème - l'imposition des limites et réglementations du PNE, ainsi que les relations interpersonnelles avec certains agents sont plus souvent rappelées. C'est donc une affaire de gestionnaires - cynégétiques ou naturalistes - qui met en porte-à-faux les scientifiques sur lesquels reposent la confirmation ou l'infirmation d'un tel effet. Des rendus objectifs sont demandés afin de vérifier les affirmations des uns et des autres.

\section{$\underline{\text { Production et emploi d'un objet-intermédiaire }}$}

Le dispositif de suivi des sangliers installé sur la commune d'Entraigues vise à fournir un objet neutre servant de support pour préciser les divergences autour des notions de «zone refuge » et d'« effet réserve ». En 2019, la mise en place de pièges photographiques le long de la limite entre la zone cœur et la zone d'adhésion au niveau du village d'Entraigues a montré qu'une partie des animaux, présents le jour dans la zone cœur du parc, en sortent la nuit vers le village d'Entraigues : plus de $75 \%$ des observations de sangliers entre $18 \mathrm{~h} 00$ et minuit sont des animaux qui sortent de la zone cœur. Le mouvement inverse est observé le matin ${ }^{8}$. L'existence d'allers-retours nocturnes entre le versant en zone cœur et la plaine du village est également démontrée par le tracé GPS des déplacements d'un mâle de $80 \mathrm{~kg}$ capturé le 6 septembre 2019. Il apparaît durant cette période que $92 \%$ des localisations de jour, mais seulement $42 \%$ des localisations de nuit se situent dans la zone cœur (Figure 2). Il a cependant été abattu le 8 décembre 2019, lors d'une de ses sorties diurnes en zone chassée, questionnant l'invulnérabilité présumées par certains acteurs - chasseurs et particuliers principalement - d'animaux fortement associés à la zone cœur. Cependant, une femelle capturée le 17 octobre 2019 n'a que $13 \%$ de ses localisations de jour et $4 \%$ de celles de nuit dans la zone cœur. Cet animal reste donc quasi-exclusivement en zone chassée, effectuant des raids nocturnes dans les prairies et cultures de la plaine et des environs du village de Valbonnais, trouvant le jour refuge dans des terrains propices à la quiétude en milieu escarpé d'altitude. Elle sera cependant tuée à la chasse moins d'un mois après avoir été capturée, le 10 novembre 2019.

Figure 2 : Suivi GPS d'un sanglier mâle sur la commune d'Entraigues (Isère, France). Sources : Données GPS, CNRS CEFE. Image Google Earth Pro 2018

\footnotetext{
${ }^{7}$ Entretien avec un ouvrier du PNE, chasseur et riverain, 2 avril 2019.

${ }^{8}$ La totalité des données durant et en dehors de la période de chasse sera analysée en 2021.
} 


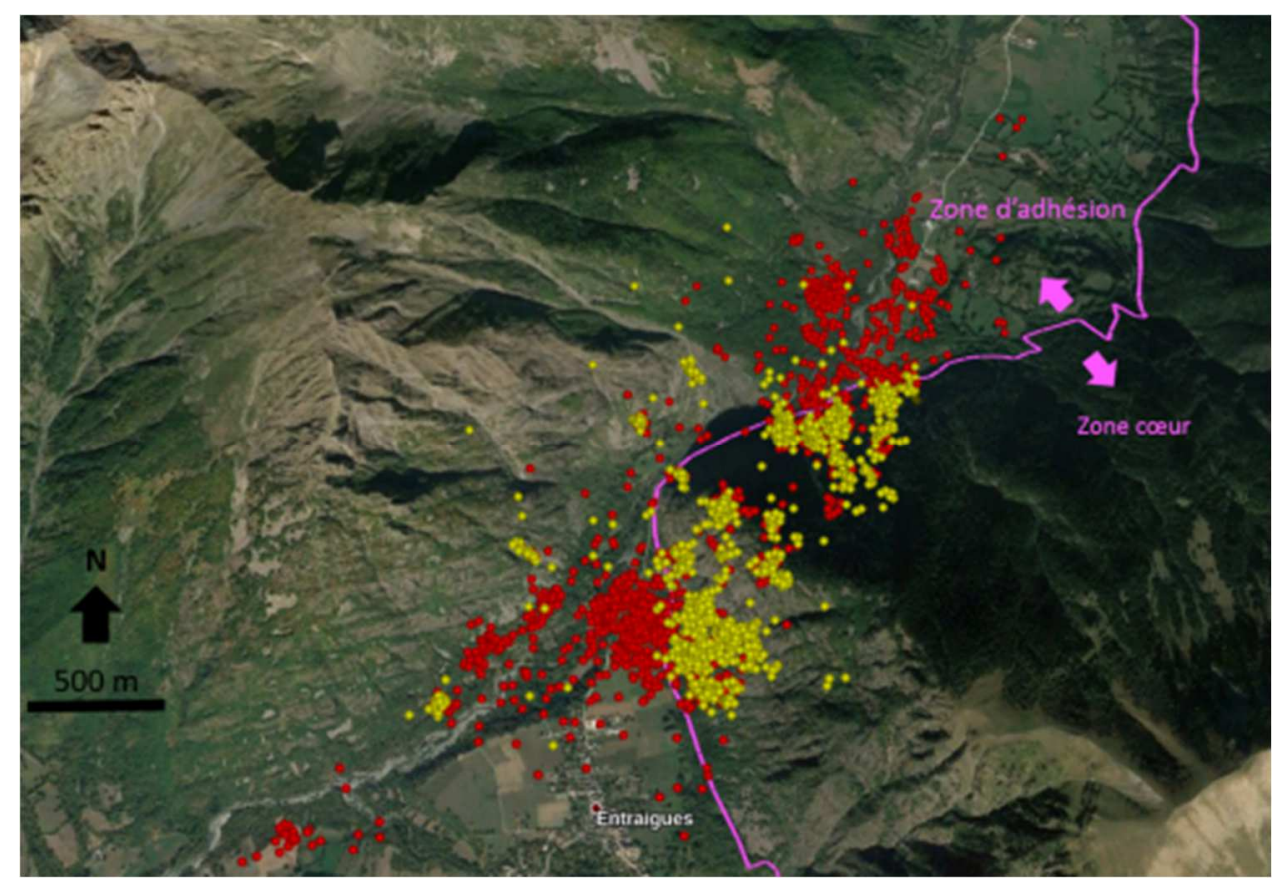

Les localisations de jour sont en jaune et de nuit en rouge. $92 \%$ des localisations de jour et $42 \%$ des localisations de nuit se situent dans la zone cœur du PNE.

Ainsi, bien que des animaux bénéficient certainement de la protection de la zone cœur, et l'exploitent activement - profitant d'un effet de réserve leur procurant un refuge qui rend plus aléatoire aux chasseurs la possibilité de tirer ces animaux - d'autres restent établis dans des zones réglementairement accessibles aux chasseurs. Par ailleurs, nombreux sont les chasseurs qui restent sensibles à l'interdiction de chasser mise en place lors de la création du PNE et le sentiment associé de mise sous tutelle écologique de leurs décisions et actions. Ils considèrent ne pouvoir réguler l'espèce en raison du refuge que génère cette interdiction sur une portion de leur ancien territoire de chasse - reproduisant par là un dispositif spatial similaire aux réserves de chasse, mais imposé et vécu comme plus rigide.

L'étude, à ce stade de sa mise en œuvre, permet de produire des preuves des déplacements et des lieux fréquentés de façon complémentaire aux indices de terrain habituels (boutis et vermillis, traces de passage des animaux etc.), mais guère de bouleverser les idées reçues et connaissances en raison de l'ancrage de l'expérience et des dires locaux au sujet du sanglier. Prêter attention aux gestes des sangliers, à leurs déplacements, permet de replacer dans le débat les suidés par l'intermédiaire de l'étude de leur comportement. Toutefois, en l'absence des chasseurs passionnés par cette espèce lors des premières réunions et ateliers, bien qu'ils y furent invités, aucun acteur n'a pris le parti de parler au nom des suidés. Si le dispositif technique apporte des indices immédiatement cartographiés et représentés, les traces et empreintes relevées sur le terrain sont autant de signes immédiatement appropriés par les acteurs, sans qu'une médiation scientifique ne soit requise. Ces-derniers méritent donc autant, sinon plus, notre attention.

\section{Prouver la présence}

La configuration du territoire de la commune d'Entraigues, la conjonction du relief et du zonage de protection font que les regards des habitants et acteurs concernés par les sangliers se portent vers les pentes au-dessus de la plaine lorsqu'il s'agit de rapporter des observations. 
Le versant fait ainsi l'objet d'une surveillance permanente, à vue ou médiatisée par des jumelles. Un réseau d'observateurs se construit, selon des groupes déjà formés et identifiés chasseurs, agents du PNE, agriculteurs, particuliers. Les chasseurs s'échangent leurs informations ; anciens chasseurs et amis ou voisins dont les jardins sont touchés font de même. Ainsi une habitante dont le jardin a été plusieurs fois touché nous rapporta qu'un voisin anciennement chasseur de sangliers et observateur régulier la prévenait par SMS en cas de présence marquante.

Les observations sont fréquentes. Les situations y conduisant sont soit provoquées, soit inattendues. Certaines, imprévues d'abord, le deviennent par la récurrence des rencontres. Aussi, chasseurs et anciens chasseurs ont le regard médiatisé par les jumelles qu'ils utilisent abondamment. Quelques-uns, d'anciens chasseurs principalement, comme cet homme âgé habitant un hameau à deux kilomètres d'Entraigues, disent observer le versant par intérêt ou comme objet de divertissement :

«Moi j'ai que ça à faire, tout l'hiver je suis là, j'ai les jumelles autour du cou, et le sanglier, des trentaines, tous les jours. [...] Parce que moi je vois... Là j'ai une longue vue, je les vois à la longue vue ${ }^{9}$. »

Ainsi il ne fut pas rare d'entendre comme une rengaine au fil des rencontres : «ils sont là », le doigt pointé vers le versant forestier. Les habitants, que leurs jardins soient touchés par des dégâts ou non, rapportent également des observations du fait de la proximité des animaux avec leur habitation. L'un d'eux, par ailleurs chasseur et agent du PNE dit en avoir vu une vingtaine une année devant son garage ; une autre, dégagée des conflits entre chasse, conservation et agriculture mais dont l'habitation donne sur le versant exposé, revient sur des individus qu'elle a dû faire fuir de son jardin, tapant sur des casseroles. Ces observations s'accompagnent de commentaires qui dépendent du contexte de celles-ci, et des effets rencontrés. Lorsque des dégâts récurrents sont constatés, c'est une demande d'intervention : « il faut faire quelque chose » répète une dizaine de fois une habitante au cours d'un entretien ${ }^{10}$; si les effets ne concernent pas l'observateur, ce sont les individus qui sont décrits : des gros, des petits rayés, leur nombre, etc. Toutes et tous s'accordent toutefois pour décrire les déplacements observés comme un aller-retour : soit que les sangliers descendent dans la plaine, soit qu'ils remontent vers le bois la surmontant. Des observations en plein jour aussi, de la part des chasseurs notamment, signalent des individus installés dans les pierriers des premières pentes, ou des « retours » dans la zone cœur au petit matin :

«Même de jour, nous ça nous est arrivé de monter au Périer [village proche, $n d l a$ ] boire le café, avant d'aller faire les pieds et que c'est jour, comme maintenant, et bah les bêtes elles passent devant et elles rentrent, ça peut être $9 \mathrm{~h}$ du matin si les bêtes elles se sont oubliées ${ }^{11}$. »

Une dernière situation enfin est mise en avant. Il s'agit des trajets effectués en voiture sur les routes des trois vallées qui entourent le village. Cette situation conduit à des considérations sur de potentielles collisions, bien qu'elles soient peu courantes dans le secteur. Selon deux particuliers, ce serait en raison de la plus grande prudence des usagers, connaisseurs des risques qu'implique la densité supposée des populations locales de sangliers. L'habitacle de la voiture constitue par ailleurs un moyen d'observation privilégié, ce qui peut paraître paradoxal

\footnotetext{
${ }^{9}$ Entretien avec un chasseur et riverain, 6 juin 2019.

${ }^{10}$ Entretien avec une riveraine, 6 juin 2019.

${ }^{11}$ Entretien avec un chasseur et riverain, 30 avril 2019.
} 
à certains naturalistes, comme ce forestier amené à intervenir dans les massifs forestiers de la commune :

«C'est malheureux pour une observation naturaliste hein, mais le plus [d'observations] c'est quand même en voiture, hors voiture c'est quand même rare ${ }^{12}$. »

Au contact direct s'ajoute l'appréhension par les traces laissées dans les cultures, sur les chemins ou les routes, au point de dessiner une « géographie de la trace » (Gagnol et Mounet, 2018, p. 14) : sentes (pistes tracées par les passages réguliers des animaux), boutis et vermillis (traces de fouissement des sangliers à la recherche de nourriture) sont abondamment commentés. De même que pour les observations directes, l'interprétation des traces est médiatisée par les effets perçus ou subis des sangliers sur soi ou autrui. Les vermillis sur les quelques terrains plats sont mis en avant par les chasseurs et particuliers dont les jardins sont touchés, minimisés par quelques agents du PNE et autres particuliers ; leur ampleur fait varier les réactions. Un site, surtout, derrière l'église du village, est mentionné dans les réponses recueillies et dans les discussions entendues. Terrain appartenant à un propriétaire peu présent sur la commune mais loué par un couple d'éleveurs, il sert d'exemple pour appuyer un discours cherchant à décrire les dégâts plus que d'objet de revendication pour le propriétaire. Objet neutre du fait de l'absence de ce dernier, il est approprié par chacun pour servir d'illustration paroxystique des effets des sangliers. Des informations complémentaires accompagnent le constat : nombre d'individus supposément coupables, la provenance de ces derniers, la fréquence de leurs ravages, etc. Pour les habitants dont le jardin est touché, ce site sert d'extension à leur surface comme à leurs revendications.

On peut noter les liens faits entre dégâts surfaciques et traces linéaires, celles marquées par des sentes reconnues. De part et d'autre de «la plaine » du village, le site de dégâts le plus visible, des zones de passage ont été mises en valeur par les enquêtés, en période de chasse comme hors de celle-ci (Figure 3).

Figure 3 : Présence et déplacements présumés des sangliers selon des chasseurs de l'ACCA d'Entraigues et déroulement typique d'une battue dans la commune d'Entraigues (Isère, France). Source : ACCA Entraigues. Image Google Earth Pro 2018

${ }^{12}$ Entretien avec un forestier et riverain, 3 avril 2019. 


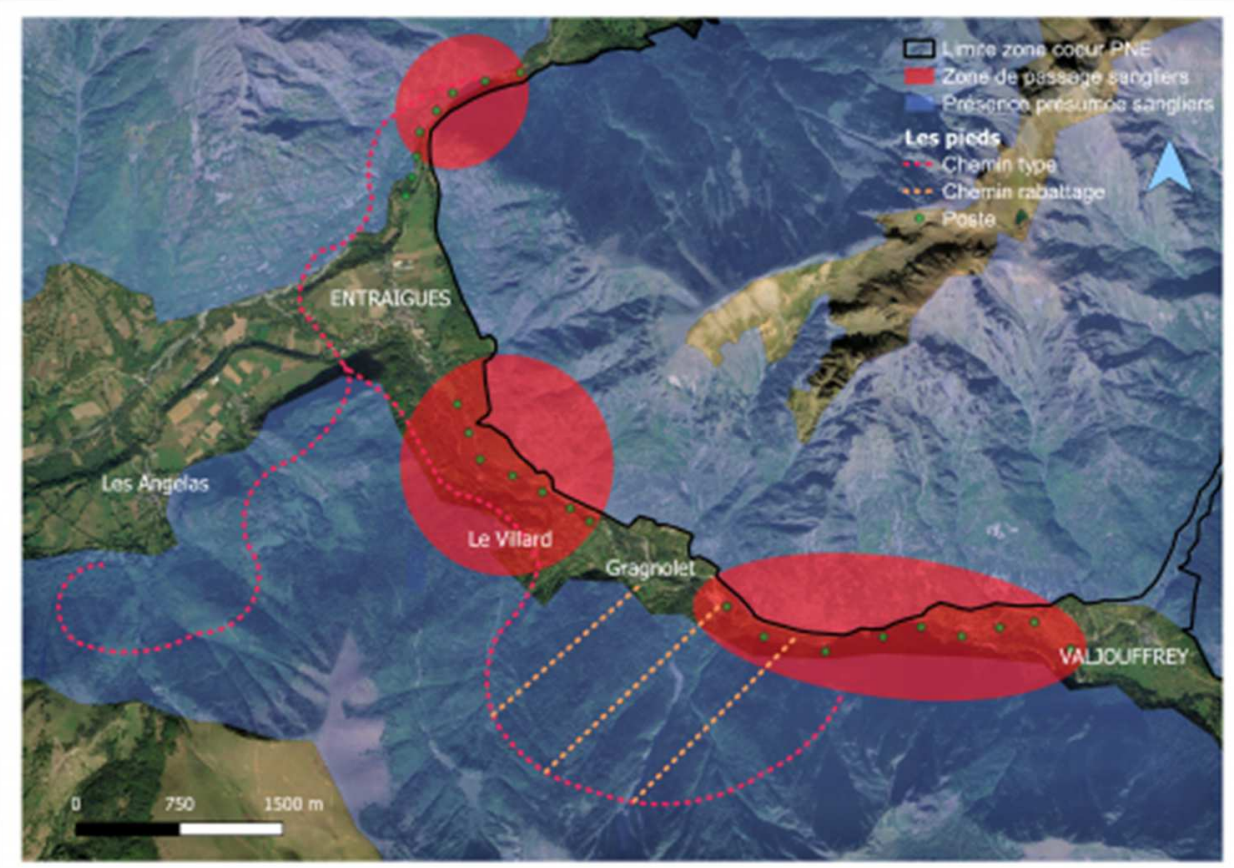

Les sangliers produisent ainsi une attention particulière des habitants à des portions spécifiques du paysage. Les lieux de leurs mobilités et de leur nourrissage sont marqués. En fonction de ces traces se forme un regard, modifié selon les intérêts économiques et symboliques des acteurs concernés et des groupes auxquels ils appartiennent. Les pratiques des groupes antagonistes sont elles aussi sujettes à une attention redoublée : les coups de fusil sont trop fréquents ou ne le sont pas assez selon que l'on déplore des dégâts ou qu'on les minimise ; on suspecte un chasseur de sortir ses chiens dans la zone cœur, malgré l'interdiction, pour déloger les sangliers qui y trouvent refuge ; on fait les comptes du tonnage de maïs utilisé pour agrainer ainsi que du nombre de sangliers abattus et selon une méthode reconnue ou dépréciée. Une éducation circonstanciée et nécessairement partiale s'élabore, et participe d'une attention renouvelée au paysage et aux usages qu'en font les acteurs identifiés comme appartenant à d'autres groupes ainsi qu'aux sangliers. Ce dernier, figé de prime abord pour l'observateur dans ses limites réglementaires, se déploie à mesure que l'on prend connaissance de l'acuité particulière de chacun. La surveillance portée aux sangliers en fait un cas exemplaire. Une approche centrée sur d'autres espèces ferait poindre d'autres géographies, fondées sur d'autres mobilités (dans cette vallée les brebis, les patous, les loups, les vautours ou les chamois par exemple, pour ce qui est des espèces les plus visibles). Les sangliers se distinguent néanmoins par le nombre d'acteurs qu'ils convoquent et les effets socioéconomiques qu'ils produisent. Une appréhension de leur comportement et de leurs déplacements, hétérogène selon les groupes et même au sein de ces derniers, est remarquable et nous a semblé constituer une dimension originale de la représentation de ce paysage et des usages qui en sont faits.

\section{Discussion : le sanglier, un animal-frontière comme clé du paysage}

Notre propos s'articule en trois points principaux. Tout d'abord, selon nous, le sanglier peut être qualifié d' « animal-frontière ». S'il se joue en effet des limites administratives, révélant et animant les controverses spatiales et économiques d'un territoire donné, il pousse également, par sa biologie, ses spatialités et ses déplacements à questionner les catégories d'analyse habituelles. Ensuite, nous évaluons pourquoi une approche à la fois interdisciplinaire et 
participative peut participer de l'élaboration d'une connaissance située de l'espèce et des enjeux socio-écologiques liés à ses déplacements. Enfin, il s'agit de définir comment le sanglier participe d'un paysage vivant - et comment par là il revivifie l'approche géographique des paysages.

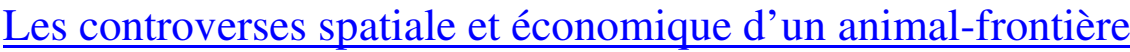

Comme nous l'avons vu plus haut, le sanglier révèle une controverse économique au sujet des dégâts agricoles. Les agriculteurs présentent ces derniers comme de fortes pertes économiques et considèrent que les indemnités allouées sont insuffisantes. Par ailleurs la lourdeur des procédures administratives freinerait les démarches de déclaration. Pour les chasseurs, les indemnités constituent indéniablement une charge financière croissante jugée excessive, ce qui pousse certains à demander aux gestionnaires d'aires protégées de prendre en charge tout ou partie du fardeau, à défaut d'autoriser la chasse dans l'espace mis en réserve. Les tensions portent également sur la justification et la mise en œuvre des pratiques d'agrainage. Le dépôt d'aliments serait perverti par certains chasseurs tout proches du site d'étude pour accroître les populations et les tableaux de chasse. Mais les tensions les plus fortes portent sans nul doute sur l'origine des animaux venant causer des dégâts.

Le parc est accusé de fournir la quiétude et l'abri nécessaire à la bonne reproduction des animaux dans sa zone cœur. Ainsi, pour beaucoup au début de cette étude, il s'agit moins de changer les pratiques de chasse et de gestion, que de permettre de chasser davantage le sanglier dans l'aire protégée voisine des lieux de dégâts. À ces considérations cynégétiques s'ajoute pour nous un questionnement épistémologique qui prend sa source dans les dires des enquêtés. Pour les sangliers, il en est des catégories comme des limites. Les suidés débordent des bornes dans lesquelles on les insère. Mieux, ils les exploitent. Difficile dès lors de reprendre la dichotomie domestique/sauvage pour les caractériser, de même que les fonctions territoriales administratives pour les lui assigner. Le sanglier reste néanmoins une espèce dite sauvage pour beaucoup d'enquêtés - le contexte géographique du territoire étudié (rural, montagnard, protégé) ne doit pas être oublié. Toutefois, la littérature et quelques remarques attestent d'une frontière facilement franchissable au sein de la famille des suidés tant biologiquement - comme l'attestent les cochons communs, le sanglier n'a pas été rétif à la domestication - qu'en fonction du comportement adopté par l'animal ou imposé à celui-ci (Pelosse et Vourc'h, 1984). Il nous reste à distinguer d'où vient l'assignation à une catégorie ou à une autre.

Bien souvent c'est la situation qui donne à l'animal son statut. Ainsi, la perception par un agent du PNE de sangliers dans leur milieu naturel ou dans un parc de chasse, aussi grand soit-il, sera différente. De même, l'appréhension des chasseurs dépend des méthodes de chasse personnellement employées et de celles des autres. Les uns dénonceront l'élevage des animaux dans l'élaboration d'un espace dédié - agrainoirs, souilles, abreuvoirs - là où les autres diront que les individus gardent leur sauvagerie dans leur capacité toujours actuelle à se défendre - ils ont, comme nous avons pu l'entendre, « du répondant ». Ainsi que Valentin Pelosse l'a résumé, l'animal cynégétique sauvage est « capable de se défendre contre son poursuivant » mais cette capacité est « un invariant instable [...] en constant réaménagement » (1993, p. 73). Les agissements de quelques acteurs témoignent d'une curiosité intense pour l'espèce, se déclinant toutefois en fonction des manières de la suivre, plus ou moins intrusives. 
Par ailleurs, si les catégories concernant les sangliers sont fluides et mouvantes, c'est, en plus de leur caractère liminaire (Donaldson et Kymlicka, 2016), leur utilisation des territoires humains qui provoque des assignations différenciées en fonction de l'espace observé. Ainsi les sangliers refoulés d'un jardin ou d'un champ ne sont en rien similaires à ceux observés incidemment sur un versant de la zone cœur du PNE, ou ceux délogés d'un fourré par des chiens de chasse. La notion de frontière est par là réinvestie, et peut-être déplacée du territoire à l'animal. Ce dernier l'incorpore et la modifie en fonction de ses actions : " Il est important de noter que ça n'est pas une caractéristique du paysage qui forme, en elle-même, une frontière. Une de ces caractéristiques ne peut devenir une frontière ou l'indice de celle-ci qu'en relation aux activités des personnes (ou animaux) pour qui elle est reconnue et vécue comme telle. » (Ingold 1993, p. 156). Le statut de l'animal produit aussi le statut de l'espace - et inversement (Mauz 2002 ; Mathevet et al. 2018). D'un jeu quasi constant avec les limites des dispositifs spatiaux et réglementaires des humains qui l'entourent ou qui lui sont assignées, le sanglier peut être qualifié «d'animal-frontière ». En rappelant aux humains ce qui dans le paysage leur échappe, les animaux, et en particulier ici le sanglier, dynamitent et dynamisent les territoires humains et participent de la vie d'un paysage social et écologique vivant, où la notion de coexistence entre les humains et la faune sauvage tend souvent, en pratique, à spécialiser l'espace et à la ségrégation spatiale (Mathevet et al., 2003). Revisiter le partage de l'espace, respecter la souveraineté des animaux sauvages sur leurs territoires, demande des dispositifs de connaissance et d'action renouvelés.

$\underline{\text { Une approche interdisciplinaire et participative pour prendre en charge l'incertitude et }}$ la diversité des légitimités d'action?

Sur le plan méthodologique, notre approche considère les déplacements des sangliers et de leurs effets socio-écologiques comme un sujet complexe. À l'incertitude causée par la difficulté d'étudier et de comprendre la mobilité animale par l'écologie, s'ajoute celle liée à l'impossibilité de prévoir les effets des interactions, ainsi que la pluralité des légitimités d'action. Dans ce contexte où la connaissance mais aussi les valeurs de la nature sont contestées, il s'agit, pour l'équipe de chercheurs impliqués dans l'étude, de co-constuire l'entendement et la définition des solutions possibles à travers un dialogue avec les partiesprenantes. Cette étude ne repose pas seulement sur la science et une approche interdisciplinaire, mais ouvre l'expertise aux savoirs locaux (Funtowicz et Ravetz, 1994), et par la mobilisation des données objectives des déplacements des animaux, tente de nourrir un dialogue local avec toutes les personnes parties prenantes du problème à résoudre. Les jeux de pouvoir sont importants à considérer (Barnaud et al., 2016) mais l'usage des cartes et photos comme objets intermédiaires est porteur d'une dynamique sociale positive bien que fragile et sensible aux positionnements stratégiques et politiques de certains acteurs.

Le suivi de deux animaux a permis de croiser données objectives et savoirs locaux pour souligner : (1) l'importance de ne pas généraliser trop hâtivement ce que font les sangliers et les raisons de leurs déplacements ; (2) comment les animaux se partagent en réalité plusieurs territoires de chasse communale et sont rebelles aux assignations territoriales humaines ; (3) comment ils ont à l'évidence leurs propres territoires de vie ; (4) comment enfin, bien que des animaux générateurs de dégâts soient souvent présentés comme difficiles à chasser, et malgré un usage intensif de la zone cœur, un animal peut être abattu en moins de quatre mois.

Finalement, au-delà de ces considérations pragmatiques, étudier la mobilité animale via une approche participative permet d'interroger la limitrophie (Derrida, 2006), soit « ce qui pousse et croit à la limite, autour de la limite, en s'entretenant de la limite, [...] ce qui nourrit la 
limite, la génère, la complique, l'élève et la complique » (p. 51), qu'il s'agisse de limites d'entités spatiales, de perceptions et de connaissances scientifiques ou empiriques. Les artefacts donnent voix à certains animaux en minimisant les effets de ventriloquie et en pénétrant la sphère politique. Les rapports aux animaux façonnent la gestion du territoire et de la faune sauvage. Il convient dès lors d'être attentif aux parcours singuliers des animaux en un paysage. Ce dernier devient d'autant plus riche à l'observateur que s'y croisent les dimensions humaines et animales de sa fabrique.

\section{D'une topographie animée à un paysage vivant}

On l'a vu plus haut, le flou catégoriel disparait quelque peu dès lors qu'il est spatialisé : c'est notamment par leur mobilité au sein d'un paysage qu'ils habitent et qu'ils fabriquent que les sangliers s'expriment. Ainsi, il convient de se défaire d'une approche uniquement territoriale propre à l'écologie spatiale pour les étudier. Si par l'intermédiaire de celle-ci des informations importantes sont délivrées, un hiatus n'est pas évité entre une approche humaine de l'espace approprié, que nous appelons territoriale, et la mobilité des animaux (Hodgetts et Lorimer, 2020) qui, bien qu'elle puisse jouer et se jouer des limites humaines, s'inscrit bien plutôt dans un paysage. Les territoires humains, matérialisés par des limites concrètes, institutionnelles ou non, sont décomposés par les spatialités des animaux non-humains.

À l'échelle de la commune d'Entraigues, des lignes (Ingold, 2013) parcourent l'espace : limite entre la zone cœur du PNE et son aire d'adhésion ; finage de la commune ; délimitations du territoire de chasse ; zone de chasse tacitement tolérée dans les premières années du PNE et matérialisée par des carrés blancs peints sur des pierres et troncs remarquables, aujourd'hui enfouis dans la végétation ou effacés, mais toujours vivaces dans les mémoires. De multiples frontières, tant sur le terrain que dans les imaginaires se sont instituées. Si les sangliers les utilisent, s'en accommodent, les humains peinent à s'en défaire - des groupes marginaux qu'il ne nous a pas été permis de rencontrer, comme les braconniers, jouent plus que d'autres de ces limites et sont pour cette raison déconsidérés légalement et délégitimés socialement. Les limites administratives marquent un paysage, le découpent en territoires distincts, redéfinissant des usages lui préexistant. Ainsi, ces lignes sont fixées pour un temps plus ou moins long, avec une possibilité d'évolution ou non, et se densifient à mesure que la mémoire épaissit. Cette conception temporelle du paysage, alliage de territoires humains en interaction avec leur milieu, nous renvoie à la pensée hétérodoxe du paysage entrevue par Georges Bertrand (1978) puis déployée par Augustin Berque (1990, 2000) ou Tim Ingold (1993 ; 2011 ; 2013) à travers la notion de «taskscape ». Pour ce dernier, un paysage n'est pas « une composition d'objets et de surfaces, mais de mouvements et de repos » (Janowski et Ingold, 2016, p. 10). Le sanglier illustre par ses déplacements incessants entre différentes portions du paysage cette approche qui, plus largement, tend à intégrer autant d'agents qu'il est d'effets.

Le mouvement animal, en questionnant spatialement des territoires administrativement figés, peut devenir une hypothèse heuristique à privilégier. Le « devenir animal » (Deleuze et Guattari, 1975) d'un paysage tient à cette perturbation : «Devenir animal, c'est précisément faire le mouvement, tracer la ligne de fuite dans toute sa positivité, franchir un seuil, atteindre un continuum d'intensités qui ne valent plus que pour elles-mêmes, trouver un monde d'intensités pures, où toutes les formes se défont, toutes les significations aussi, signifiants et signifiés, au profit d'une manière non-formée, de flux déterritorialisés, de signes asignifiants. » (ibid., p. 24) Par leur incessante mobilité, les animaux signent le paysage qu'ils habitent de leurs traces, se défont des territoires humains ou font avec ces derniers. Les considérer dans notre approche d'un espace donné ne peut que complexifier et densifier toute 
conception paysagère. Si les sangliers, par leurs effets peuvent compliquer des situations déjà tendues (la condition paysanne actuelle notamment) ou participer de relations sociales conflictuelles (entre chasseurs et gestionnaires d'aires protégées par exemple), ils permettent de porter de nouveau attention aux temporalités propres au paysage, aux mouvements qui le parcourent, humaines et non-humaines.

\section{Conclusion}

La coexistence des sangliers, ou plus généralement des animaux sauvages dans l'espace approprié par les humains nécessite d'explorer les modalités d'une cohabitation : comment vivre avec ou malgré l'autre ? À l'issue de cette première étape, le sanglier révèle comment le partage de l'espace ne va pas sans conflit et sans questionner l'identité des sujets et objets, sinon des lieux. Tantôt ressource et gibier, tantôt patrimoine naturel, risque ou opportunité, le sanglier permet de repenser les rapports homme-milieu. L'écologie spatiale renouvelle les apports de la biologie animale, de l'écologie de la conservation mais aussi de la géographie animale au-delà des questions habituelles liées aux représentations sociales et aux modalités d'exploitation de la nature et de l'espace. Croiser les disciplines s'est avéré déterminant. Le sanglier montre que les animaux permettent de porter l'analyse de l'évolution des territoires et des activités humaines dans le contexte de l'Anthropocène et de l'enfrichement, voire l'« ensauvagement » de certaines régions rurales (Schnitzler et Génot 2020). Il contribue à interroger les dimensions culturelles, sociales et politiques mais aussi écologiques des maux environnementaux de nos sociétés. Au-delà des dimensions strictement écologique et cynégétique, la géographie animale que nous déployons ici, à partir des objets intermédiaires que sont les tracés GPS et pièges photographiques, permet de mettre les animaux au centre de l'analyse et de revisiter les questions éthologiques, anthropologiques et philosophiques, mais aussi géopolitiques et stratégiques des jeux d'acteurs qui se déploient dans la gestion de l'espace comme de l'espèce. Le sanglier a été façonné et continue de l'être par les activités humaines, directement et indirectement, mais il façonne aussi le paysage écologique et politique qu'il occupe. La géographie animale est plus que jamais nécessaire au moment où les changements environnementaux planétaires et les politiques de conservation de la biodiversité sont amenés à évoluer sensiblement.

\section{Bibliographie}

BALLARI S.A., BARRIOSGARCÍA M.N., 2014, A Review of Wild Boar Sus Scrofa Diet and Factors Affecting Food Selection in Native and Introduced Ranges, Mammal Review, vol. $44, \mathrm{n}^{\circ} 2$, p. 124-134.

BARNAUD C., D’AQUINO P., DARE W. et al., 2016, Dispositifs participatifs et asymétries de pouvoir : expliciter et interroger les positionnements, Participations, vol. $16, \mathrm{n}^{\circ} 3$, p. 137-166.

BARUA M., 2014, Bio-Geo-Graphy: Landscape, Dwelling, and the Political Ecology of Human-Elephant Relations, Environment and Planning D: Society and Space, vol. 32, ${ }^{\circ} 5$, p. 915-934.

BARUA M., SINHA A., Animating the urban: an ethological and geographical conversation, Social \& Cultural Geography, vol. 20, n 8, p. 1160-1180.

DOI : $\underline{10.1080 / 14649365.2017 .1409908}$ 
BAUBET E., 1998, Biologie du sanglier en montagne : biodémographie, occupation de l'espace et régime alimentaire, thèse de doctorat en biologie des populations, Université Lyon 1. $348 \mathrm{p}$.

BEAR C., 2011, Being Angelica? Exploring Individual Animal Geographies, Area, vol. 43, $\mathrm{n}^{\circ} 3$, p. 297-304.

DOI : $\underline{10.1111 / \mathrm{j} .1475-4762.2011 .01019 . \mathrm{x}}$

BECK C., FABRE E., 2010, Interroger le loup historique ? Entre la biologie et l'histoire : un dialogue interdisciplinaire, in MORICEAU J-M., MADELINE P. (dir.), Repenser le sauvage grâce au retour du loup. Les sciences humaines interpellées, Caen, MSH, p. 13-21.

BENHAMMOU F., 2007, Crier au loup pour avoir la peau de l'ours : une géopolitique locale de l'environnement à travers la gestion et la conservation des grands prédateurs en France, thèse de doctorat en géographie, Paris, ENGREF, 655 p.

BENHAMMOU F., 2016, Une histoire contemporaine de la géographie française de l'animal, in CHARTIER D., RODARY E. (dir.), Manifeste pour une géographie environnementale, Paris, Presses de Sciences Po, p. 141-164.

BERQUE A., 2000, Écoumène : introduction à l'étude des milieux humains, Paris, Belin, $446 \mathrm{p}$.

BERQUE A., 1990, Médiance : de milieux en paysages, Montpellier, GIP Reclus, 222 p.

BERTHIER A., CLERGEAU P., RAYMOND R., 2017, De la belle exotique à la belle invasive : perceptions et appréciations de la Perruche à collier (Psittacula krameri) dans la métropole parisienne, Annales de géographie, vol. 716, n 4, p. 408-434.

DOI : $10.3917 /$ ag. 716.0408

BERTRAND G., 1978, Le paysage entre la Nature et la Société, Revue géographique des Pyrénées et du Sud-Ouest. Sud-Ouest Européen, vol. 49, n² 2, p. 239-258.

BLANC N., COHEN M., 2002, L'animal : une figure de la géographie contemporaine, Espaces et sociétés, vol. 110-111, n 3, p. 25-40.

DOI : $10.3917 /$ esp.g2002.110-111.0025

BOBEK B., FURTEK J., BOBEK J. et al., 2017, Spatio-temporal characteristics of crop damage caused by wild boar in north-eastern Poland, Crop Protection, vol. 93, p. 106-112. DOI : $\underline{\text { 10.1016/j.cropro.2016.11.030 }}$

BORTOLAMIOL S., RAYMOND R., SIMON L., 2017, Territoires des humains et territoires des animaux : éléments de réflexions pour une géographie animale, Annales de géographie, vol. $716, \mathrm{n}^{\circ} 4$, p. 387-407.

DOI : $10.3917 /$ ag. 716.0387

BOUSSIN L., 2008, L'animal sauvage à travers ses représentations, l'exemple du chevreuil en Limousin, Annales de géographie, vol. 663, n 5, p. 48-61. 
BULLER H., 2014, Animal Geographies I, Progress in Human Geography, vol. 38, ${ }^{\circ} 2$, p. 308-318.

BULLER H., 2015, Animal Geographies II: Methods, Progress in Human Geography, vol. $39, n^{\circ} 3$, p. 374-384.

BULLER H., 2016, Animal Geographies III: Ethics, Progress in Human Geography, vol. 40, $\mathrm{n}^{\circ} 3$, p. 422-30.

CARDOUX J-N., PEREA A., 2019. Restaurer l'équilibre agro-sylvo-cynégétique pour une pleine maîtrise des populations de grand gibier et de leurs dégâts à l'échelle nationale. Mission parlementaire, Paris, Sénat, Assemblée Nationale, 80 p.

CARNIS L., FACCHINI F., 2012, Une approche économique des dégâts de gibier.

Indemnisation, prix et propriété ». Économie rurale. Agricultures, alimentations, territoires, $\mathrm{n}^{\circ} 327-328$, p. 126-142.

CHANDELIER M., 2018, Le loup dans la presse française contemporaine : analyse des fonctionnements argumentatifs médiatiques, thèse doctorat en sciences du langage, Université Montpellier 3, $328 \mathrm{p}$.

CHARLEZ A., 2008, Le sanglier, le droit et l'indemnisation des dégâts, Faune Sauvage, $\mathrm{n}^{\circ} 282$, p. $51-58$.

DELEUZE G., GUATTARI F., 1975, Kafka : pour une littérature mineure. Paris, Éditions de Minuit, $160 \mathrm{p}$.

DERRIDA J., 2006, L’animal que donc je suis, Paris, Galilée, 218 p.

DONALDSON S., KYMLICKA W., 2016, Zoopolis : une théorie politique des droits des animaux, Paris, Alma éditeur, $400 \mathrm{p}$.

FATTEBERT J., BAUBET E., SLOTOW R., et al., 2017, Landscape Effects on Wild Boar Home Range Size under Contrasting Harvest Regimes in a Human-Dominated AgroEcosystem, European Journal of Wildlife Research, vol. 63, $\mathrm{n}^{\circ}$ 2, p. 32.

DOI : $10.1007 / \mathrm{s} 10344-017-1090-9$

FORSYTH I., 2016, A Bear's Biography: Hybrid Warfare and the More-than-Human Battlespace, Environment and Planning D: Society and Space, vol. 35, n 3, p. 495-512.

FUNTOWICZ S.O., RAVETZ J.R., 1994, Uncertainty, Complexity and Post-Normal Science, Environmental Toxicology and Chemistry, vol. 13, n 12 , p. 1881-1885.

GAGNOL L., MOUNET C., ARPIN I., 2018, De la piste animale aux lignes de désir urbaines. Une approche géoichnologique de la trace, L'Information géographique, vol. 82, $\mathrm{n}^{\circ} 2$, p. 11-38.

GIBBS L.M., 2020a, Animal Geographies I: Hearing the Cry and Extending Beyond, Progress in Human Geography, vol. 44 n 4, p. 769-777. 
GIBBS L., 2020b, Animal Geographies II: Killing and Caring (in Times of Crisis), Progress in Human Geography, 1st published online July 21, 2020, [en ligne].

GIREL J., 2006, Quand le passé éclaire le présent : écologie et histoire du paysage, Géocarrefour, vol. 81, n 4, p. 249-64.

HELL B., 1998, Sang noir : chasse, forêt et mythe de l'homme sauvage en Europe. Paris, Flammarion, $381 \mathrm{p}$.

HODGETTS T., LORIMER J., 2020, Animals' Mobilities, Progress in Human Geography, vol. 44, n 1 , p. 4-26.

HOVORKA A.J., 2017, Animal Geographies I: Globalizing and Decolonizing, Progress in Human Geography, vol. 41, n 3, p. 382-394.

HOVORKA A.J., 2018, Animal Geographies II: Hybridizing, Progress in Human Geography, vol. 42, n 3, p. 453-462.

HOVORKA A.J., 2019, Animal Geographies III: Species Relations of Power, Progress in Human Geography, vol. 43, n 4, p. 749-757.

INGOLD T., 2011, Being alive: essays on movement, knowledge and description, London, Routledge, $288 \mathrm{p}$.

INGOLD T., 1993, The temporality of the landscape, World Archaeology, vol. 25, $\mathrm{n}^{\circ} 2$, p. $152-174$.

INGOLD T., 2013, Une brève histoire des lignes. Bruxelles, Zones sensibles, 269 p.

JANOWSKI M., INGOLD M. (Dir.), 2016, Imagining landscapes: past, present and future, London, Routledge, 184 p.

JOHNSTON C., 2008, Beyond the Clearing: Towards a Dwelt Animal Geography, Progress in Human Geography, vol. 32, n 5, p. 633-649.

LE LAY Y.F., ARNOULD P., COMBY E., 2017, Le castor, un agent en eau trouble. L'exemple du fleuve Rhône, Géocarrefour, vol. 91, n 4, [en ligne].

LOMPECH M., RICARD D., RIEUTORT L., 2018, L'âne en France, ses usages et ses territoires, Géocarrefour, vol. 92, n 3, [en ligne].

LORIMER H., 2006, Herding Memories of Humans and Animals, Environment and Planning D: Society and Space, vol. 24, $\mathrm{n}^{\circ} 4$, p. 497-518.

LORIMER J., SRINIVASAN K., 2013, Animal geographies, in JOHNSON N.C., SCHEIN R.H., WINDERS J. (dir.), The Wiley-Blackwell Companion to Cultural Geography, Oxford, Wiley \& Sons, p. 332-342.

MARCHAND G., 2013, Les conflits hommes/animaux sauvages sous le regard de la géographie, Carnets de géographes, $\mathrm{n}^{\circ} 5$, [en ligne]. 
MASSEI G., GENOV P.V., 2004, The environmental impact of wild boar, Galemys, vol. 16, $\mathrm{n}^{\circ} 1$, p. 135-145.

MASSEI G., KINDBERG J., LICOPPE A., et al., 2015, Wild Boar Populations up, Numbers of Hunters down? A Review of Trends and Implications for Europe, Pest Management Science, vol. 71, $\mathrm{n}^{\circ}$ 4, p. 492-500.

DOI : $\underline{10.1002 / p s .3965}$

MATHEVET R., BECHET A., 2020, Politiques du flamant rose, Vers une écologie du sauvage, Marseille, WildProject, 200 p.

MATHEVET R., BOUSQUET F., LE PAGE C., et al., 2003, Agent-based simulations of interactions between duck population, farming decision and hunting leasing in the Camargue (Southern France), Ecological Modelling, vol. 165, p. 107-126.

MATHEVET R., GODET L. (Dir.), 2015, Pour une géographie de la conservation. Biodiversités, natures et sociétés, Paris, L'Harmattan, 404 p.

MATHEVET R., OLIVIER A., BECHET A., 2018, Nuisibles d'hier et d'aujourd'hui. Le lapin, le sanglier et le flamant dans la réserve de biosphère de Camargue, in LUGLIA R., (Dir.), Sales bêtes ! Mauvaises herbes ! " Nuisibles », une notion en débat, Rennes, PUR, p. 173-187.

MAUZ I., 2002, Les conceptions de la juste place des animaux dans les Alpes françaises, Espaces et sociétés, vol. 110-111, n 3, p. 129-146.

MORELLE K., PODGÓRSKI T., PRÉVOT C., et al., 2015, Towards Understanding Wild Boar Sus Scrofa Movement: A Synthetic Movement Ecology Approach, Mammal Review, vol. $45, \mathrm{n}^{\circ} 1$, p. 15-29.

MOUNET C., 2011, Conflits et reconfigurations socio-spatiales autour du sanglier : des postures générales aux arrangements locaux en territoire rural, Economie Rurale, ${ }^{\circ} 327-328$, p. 79-95.

MOUNET C., 2007a, Les territoires de l'imprévisible. Conflits, controverses et "vivre ensemble" autour de la gestion de la faune sauvage. Le cas du loup et du sanglier dans les Alpes françaises, thèse de doctorat de géographie, Université Grenoble I, 565 p.

MOUNET C., 2007b, Vivre avec des animaux «à problème ». Le cas du loup et du sanglier dans les Alpes françaises, Revue de Géographie Alpine, vol. 96, n 3, p. 55-64.

NATHAN R., GETZ W.M., REVILLA E., et al., 2008, A movement ecology paradigm for unifying organismal movement research, PNAS, vol. 105, n 49, p. 19052-19059.

DOI : $10.1073 /$ pnas.0800375105

OLIVIER DE SARDAN J.-P., 2004, La rigueur du qualitatif. L'anthropologie comme science empirique, Espace Temps, vol. 84, n 1 , p. 38-50.

PELOSSE V., 1993, Construction de l'animal cynégétique. Mouflons et sangliers en Languedoc, Études rurales, vol. 129, n 1 , p. 73-82. 
PELOSSE V., VOURC'H A., 1982, Chasse au sanglier en Cévennes, Études rurales, vol. 87 , $\mathrm{n}^{\circ} 1$, p. 295-307.

PELOSSE V., VOURC'H A., 1984, Un jeu avec le sanglier, Ethnologie française, vol. 14, $\mathrm{n}^{\circ} 4$, p. 343-54.

PHILO C., WILBERT C. (Dir.), 2000, Animal spaces, beastly places: new geographies of human-animal relations. London, Routledge, 336 p.

PNMS, 2009, Plan national de maîtrise du sanglier. Un cadre d'actions techniques pour agir au plan départemental, Paris, Ministère de l'Écologie, du Développement Durable et de la Mer, $25 \mathrm{p}$.

QUIRÓS-FERNÁNDEZ F., MARCOS J., ACEVADO P., et al., 2017, Hunters Serving the Ecosystem: The Contribution of Recreational Hunting to Wild Boar Population Control, European Journal of Wildlife Research, vol. 63, $\mathrm{n}^{\circ} 3, \mathrm{p} .57$.

DOI : $10.1007 / \mathrm{s} 10344-017-1107-4$

RAYNAL J.-C., 2004, Pratiques cynégétiques, transformations territoriales et régulations sociales : vers la mise en place d'une gestion concertée des populations de sangliers dans le Sud de la France, thèse de doctorat de géographie, Université Montpellier 3, 429 p.

ROSE M., WYLIE J., 2006, Animating Landscape, Environment and Planning D: Society and Space, vol. 24, $\mathrm{n}^{\circ}$ 4, p. 475-479.

SALDAQUI F., 2013, Configurations territoriales et gestion de la faune sauvage : les sangliers aquitains entre espaces protégés et campagnes chassées, thèse de doctorat de géographie, Université de Pau et des Pays de l'Adour, 347 p.

SCHNITZLER A., GENOT J-C., 2020, La nature férale ou le retour du sauvage : pour l'ensauvagement de nos paysage. Genève, Jouvence, 175 p.

SEYMOUR M., WOLCH J., 2010, «A Little Bird Told Me... »: Approaching Animals Through Qualitative Methods, in DELYSER D., HERBERT S., AITKEN S., CRANG M., McDOWELL L. (Dir.), The Sage Handbook of Qualitative Geography, London, Sage Publications, p. 305-320.

STAR S.L., GRIESEMER J.R., 1989, Institutional Ecology, 'Translations' and Boundary Objects: Amateurs and Professionals in Berkeley's Museum of Vertebrate Zoology, 1907-39, Social Studies of Science, vol. 19, $\mathrm{n}^{\circ} 3$, p. 387-420.

THRIFT N., DEWSBURY J-D., 2000, Dead Geographies - And How to Make Them Live, Environment and Planning D: Society and Space, vol. 18, n 4, p. 411-443.

THURFJELL H., SPONG G., ERICSSON G., 2013, Effects of hunting on wild boar Sus scrofa behaviour, Wildlife Biology, vol. 19, $\mathrm{n}^{\circ}$ 1, p. 87-93.

TOÏGO C., SERVANTY S., GAILLARD J-M., et al., 2008, Disentangling Natural From Hunting Mortality in an Intensively Hunted Wild Boar Population, Journal of Wildlife Management, vol. 72, $\mathrm{n}^{\circ}$ 7, p. 1532-1539. 
TOLON V., DRAY S., LOISON A., et al., 2009, Responding to spatial and temporal variations in predation risk: space use of a game species in a changing landscape of fear, Canadian Journal of Zoology, vol. 87, $\mathrm{n}^{\circ}$ 12, p. 1129-1137.

DOI : $10.1139 / Z 09-101$

TOLON V., BAUBET E., 2010, L'effet des réserves sur l'occupation de l'espace par le sanglier, Faune Sauvage, ${ }^{\circ} 288$, p. 14-18.

TUCKER M.A., BÖHNING-GAESE K., FAGAN W.F., et al., 2018, Moving in the Anthropocene: Global Reductions in Terrestrial Mammalian Movements, Science, vol. 359, $\mathrm{n}^{\circ}$ 6374, p. 466-469.

DOI : $\underline{10.1126 / \text { science.aam9712 }}$

VALLEE M., LEBOURGEOIS F., BAUDET E., et al., 2016, Le sanglier en Europe : une menace pour la biodiversité ? Revue Forestière Française, vol. 68, n 6, p. 505-518.

DOI : $\underline{10.4267 / 2042 / 62398}$

VETTER S.G., RUF T., BIEBER C., et al., 2015, What Is a Mild Winter? Regional Differences in Within-Species Responses to Climate Change, PLOS ONE, vol. 10, $\mathrm{n}^{\circ} 7$, e0132178.

DOI : $\underline{10.1371 / \text { journal.pone. } 0132178}$

VINCK D., 1999, Les objets intermédiaires dans les réseaux de coopération scientifique :

Contribution à la prise en compte des objets dans les dynamiques sociales ». Revue française de sociologie, vol. $40, \mathrm{n}^{\circ} 2$, p. 385-414.

WHATMORE S., 2002, Hybrid geographies : natures, cultures, spaces. London, SAGE, $201 \mathrm{p}$.

WHATMORE S., 2006, Materialist returns: practicing cultural geography in and for a morethan-human world, Cultural Geographies, vol. 13, n 4, p. 600-609.

DOI : $\underline{10.1191 / 1474474006 c g j 377 \text { oa }}$

WOLCH J.R., EMEL J. (Dir.), 1998, Animal geographies: place, politics, and identity in the nature-culture borderlands. London, Verso, $310 \mathrm{p}$.

Auteurs

\section{$\underline{\text { Roméo Bondon }}$}

École Normale Supérieure de Lyon, Lyon, France romeo.bondon@ens-lyon.fr

\section{$\underline{\text { Raphaël Mathevet }}$}


CEFE, Univ. Montpellier, CNRS, EPHE, IRD, Université Paul Valéry Montpellier 3, Montpellier, France et Institut Français de Pondichéry, UMIFRE 21CNRS, MAE,

Pondichéry, Inde. raphael.mathevet@cefe.cnrs.fr

\section{Coralie Mounet}

Univ. Grenoble Alpes, CNRS, Sciences Po Grenoble, Pacte, 38000 Grenoble, France coralie.Mounet@univ-grenoble-alpes.fr

\section{Simon Chamaillé-Jammes}

CEFE, Univ. Montpellier, CNRS, EPHE, IRD, Université Paul Valéry Montpellier 3 simon.chamaille@cefe.cnrs.fr

Référence :

Roméo Bondon, Raphaël Mathevet, Coralie Mounet et Simon Chamaillé-Jammes, «Passer les limites, rythmer le territoire. Paysage et mobilités du sanglier en Valbonnais (Isère, France) », Géocarrefour [En ligne], 95/4 | 2021, mis en ligne le 29 janvier 2021, consulté le 20 octobre 2021. URL : http://journals.openedition.org/geocarrefour/16917 ; DOI : https://doi.org/10.4000/geocarrefour.16917 